\title{
Life within chronic care: is this a service or sentence?
}

\author{
Pragashnie Govender, Jacqui Boyd, Aadila Hassim, Tamryn Jordaan, \\ Nazmeera Mahomed, Daniela Straeuli-Paul
}

Discipline of Occupational Therapy, School of Health Sciences, University of KwaZulu-Natal (Westville campus) Private Bag x 54001, Durban4000

\begin{abstract}
Background: Understanding the meaning of one's lived experiences improves one's understanding of what it means to be human, in association with the social, cultural and historical context in which being a human occurs. The authors in this study describe the lived experiences of residents within a chronic care facility including the practitioner and family perspectives.

Methods: A qualitative approach was employed with a single-site, descriptive, instrumental case study design. Purposive sampling was utilised to select the chronic facility. Multiple sources of evidence included narratives, semi-structured interviews with staff, residents and family members, and activity profiles of the residents augmented by an ergonomic evaluation of the facility. Content analysis using within-case analysis was implemented.

Results: The greatest impact on the quality of the resident's lived experiences emanates from the physical, organisational and social environments in which they reside. Limited resources, poor staff attitudes and routines that are enforced both on the staff of the facility and the residents appear to reduce optimal functioning within the facility. Furthermore the residents' intrinsic motivation, presence of enforced idleness, learned helplessness and institutionalisation is often intensified and becomes characteristic of the residents lived experiences.

Discussion: The lived experiences of the residents are described according to quality of life indicators that were identified during the study and supported by literature. These include the influence of the physical, social and organizational environments on the residents' functional status, which comprises physical well-being and emotional well-being and engagement in meaningful occupations including social interaction and relationships with other individuals.

Conclusions: Findings of this study may be valuable in understanding and facilitating a positive change in service delivery within chronic care centres.
\end{abstract}

Keywords: chronic care, institutionalisation, lived experiences, residents, occupational deprivation

DOI: http://dx.doi.org/10.4314/ahs.v15i2.46

\section{Background}

The study of lived experiences allows for a deeper understanding of the "nature and meaning" of normal, daily experiences ${ }^{1}$. It provides conceivable insights into life that allows individuals to become more conscious of the world around them and their place within it. Individuals who suffer from a chronic disease define their

\author{
Corresponding author: \\ Pragashnie Govender \\ Discipline of Occupational Therapy, \\ School of Health Sciences, \\ University of KwaZulu-Natal \\ (Westville campus) \\ Private Bag x 54001, Durban4000 \\ Cell: 0716056931 \\ E-mail: naidoopg@ukzn.ac.za
}

condition in terms of their lived experience and health on a day to day knowledge, instead of perceiving their disease within a "biomedical" approach. ${ }^{2}$

Many individuals with chronic diseases are often placed in chronic care facilities, which cater for their medical needs, and often are as a result of family members not being able to sufficiently care for these individuals at home. This inevitably influences the care that patients receive in their immediate and home environments, in which they are unable to be adequately accommodated for. Chronic care facilities thus ensure that these individuals are able to receive the medical care and rehabilitation that is necessary for maximal functioning and maintenance, whilst concurrently considering possible and aggravating family limitations.

\section{Chronic care facilities in South Africa}

Chronic or long term care in South Africa is promoted 
through public and private facilities. These enable inavailable as needed by each resident, and the

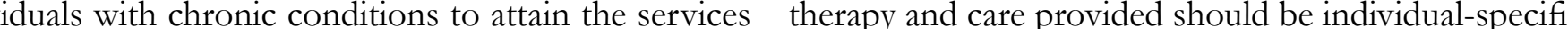
that they require by promoting functional abilities and so as to accommodate the unique qualities, strengths capacities. Such institutions provide residents with food and needs of each resident. and medical care, as well as structured activities that influence a daily routine. Rehabilitation services that are The authors in this study sought out to describe the regularly provided in such facilities include occupation- lived experiences of residents living within a chronic al therapy, speech-language therapy and physiotherapy, care facility, including the practitioner and family perdepending on the nature of the chronic care facility and spectives, in order to highlight some of the challenge the population catered for, while services such as social as experienced by these consumers of the service. work may also be offered.

Long term facilities are primarily involved in addressing the physical well-being of their population, and thus their population's psychological well-being may be overlooked. This results in a decrease in independence and control of their primary activities. ${ }^{3}$ The deficiency in independence and control results in a loss of role duties with dependency and reliance on staff for basic requirements.

\section{Ethical Considerations for Service Provision in}

\section{Chronic Care}

Ethically, chronic care should provide the individual residents with respect and dignity whilst their needs are being addressed. Health care should be accessible, efficient and affordable for all, and individuals working within this sector should take responsibility and account for interventions/service delivery within the institution. The systems should not place unbearable strain on the families whilst simultaneously allowing individuals to lead independent lives as far as possible. ${ }^{4}$

Good practice within chronic care does not only reflect the adherence to the Patients' Rights Charter ${ }^{5}$ and Batho Pele policy, ${ }^{6}$ but also ensuring that the quality of life of the residents within chronic care facilities is upheld as much as is possible within the physical and financial environmental constraints of such facilities. In orde to achieve this, chronic care facilities should encourage the engagement of residents in meaningful occupations, which has an important role in the achievement of well-being and personal satisfaction ${ }^{7}$. The family members of residents residing in chronic care facilities should be included in the care, with active involvement in decisions taken for their relative's well-being. Additionally, residents of chronic care facilities should be offered treatment and care on trans- and multi-disciplinary levels, whereby nursing, rehabilitation and social

\section{Methods}

A single-site, descriptive, instrumental case study design within a qualitative approach was used. Ethical clearance and gatekeeper permission from the hospital manager (CEO) of the setting was obtained in addition to informed consent from all participants.

Purposive sampling was utilised to select the chronic facility. For the purpose of obtaining accurate, rich data, multiple sources of evidence were utilised, namely narratives (4 residents), semi-structured interviews with staff ( 4 rehabilitation staff, 6 nursing staff), residents ( activity profiles (8 profiles) of the residents augmented by a comprehensive ergonomic evaluation of the facility (via measurements and photographs).

Content analysis, using within-case analysis was implemented on three levels and was used to organise the information into core themes. Reduction of researche bias and trustworthiness throughout all phases of the study was ensured through investigator and data triangulation, use of multiple sources of data, construction of a chain of evidence and pattern matching of data during analysis.

\section{Results}

Within the chronic care institution, residents' affect or mood, levels of motivation and capacity for occupational engagement is often influenced by the structure of the facility and the features of everyday life that residents experience.

The findings reflect that the greatest impact on the quality of the residents' lived experiences emanate from the environment in which they reside. Three levels of environmental barriers were identified by all participants.
Physical barriers included the accessibility of services and the manner in which residents mobilise themselves. "...this moment in time, I am stuck in a coffin that has not (got) any breaks now...it's got no bearings...it's a mess..." (Anna-Resident) (Elaine-Family disadvantage in terms of being able to receive and have Member)

access to resources" (Thando-Rehabilitation Professional)

“...a lot of patient's are disadvantaged in that regard and a lot more patients could do with individual therapy..

"It's built on a hill so patients, often patients who can was there more time and professionals available" (Senself-propel still need somebody to port them as there is a zo-Family Member)

massive hill leading up to the department" (Mark-Family Member)

“...I'm doing three jobs at the moment...So, I'm very... very busy so... I do what I have to do with the patients,

Social \& organisational barriers include the routines but I can't offer extra because there's just a shortage of and structures within the hospital, as well as the atti- staff" (Patricia-Nursing Professional)

tudes of staff.

“...well the nurses they look at you like you just crawled out a... gorgonzola cheese” (Mary-Resident)

The study further illustrated that the residents' intrinsic motivation is another factor influencing their lived experiences.

"And they come and give you another drug....and you're drugged out - you are so bloody stoned it's scary..." (Philip-Resident)

". a lot of it comes from individual motivation....and I think due to institutionalisation that declines as they stay here - it progresses...” (Rehabilitation Professional)

"...they like their system and then they not prepared "I think it's difficult with being in a chronic care facilito look at the system and readjust to anyone...in par- ty whether you work here or whether you staying here ticular patients' desire and requirement" (Elaine-Family Member)

“...you feel like you're a prisoner...” (Sipho-Resident)

Family member opinions are highlighted below.

"...they have a predisposed concept of what they believe...(we) should be doing" (Elaine)

"...fire all the staff and replace with people...that reall want to help..." (Thembi)

"...some of them are very nasty to the patients". (Sen-

"...they doing their job... don't ask them to do anything else slightly out of the way..." (Mark)

Consequently, effective service provision is limited and so is functional improvement of the residents. This phenomenon, in effect, serves to hinder residents' well-being and challenges the ethical principles that are associated with service provision. it's....that... feeling of the sameness...I don't know if it's a healthy environment to work at in the...in the longrun...I think it's really difficult to keep motivated and... I think it's the same for the staff and the patients alike" (Hannah-Rehabilitation Professional)

As individual's intrinsic motivation and volition diminishes and/or weakens, the phenomena of learned Singh" further proposes a lack of autonomy as a result infexibility and rigid routines, known as "routinisation", which increases their susceptibility to learned helplessness. Additionally, the enforced culture of dependency fostered through the organisational and physcal environment of the institution further compounded the development of attitudes congruent with learned helplessness which, when intensified, may progress into institutionalisation. This was evident in resident attitudes towards life.

"So, when I visit her, she asks me to do it for (assist) her. I get so disappointed..." (Elaine-Family member) helplessness and institutionalisation are strengthened. 
'Well it doesn't really worry me. You know...I have been here three years now... I have got used to what's going on around me..." (Anna-Resident)

Through the development of learned helplessness, the resident's personalities and individuality are moulded to the structure and routines of the facility, rather than individualized and specific. This presence of enforced idleness, learned helplessness and institutionalisation, is often intensified, and becomes a characteristic of the residents lived experiences within the facility.

....we stuck onto a conveyor belt and... we just go along and do what they require us to do" (Phillip-Resident)

Lifestyles congruent with occupational deprivation and occupational imbalance, often becomes an enforced and natural phenomenon of the facility, compounded and reinforced by both the residents, the staff and the resources therein.

“...a lot of them just...sit on their own and stare into space (laugh) with like no stimulation...”'(Senzo-Family Member)

"I think boredom's a big one... and boredom perpetuates boredom...”'(Elaine-Family Member)

The emotional well-being of the residents is furthe impacted by physical disabilities which are often associated with loss of control of basic bodily functions. Consequently, residents' emotions are often subjected and reduced to humiliation and feelings of degradation. This inevitably impacts their self-concept, and often imposes occupational dysfunction and apathy. Residents were quoted as saying,

“...well, you've got a crap in the nappy...it's so humiliating, degrading..." (Anna)

“...my daughter found a dead fly in my hair..." (Mary)

In contrast, one cannot mitigate the benefits that are associated with chronic care facilities as the basic needs of the residents are provided to those individuals whose families are unable to care for them with the require level of skill. Additionally, residents within a chronic care facility are afforded the opportunity for contact and socialisation.

Predetermined routines and schedules allows for the uninterrupted and fluent orchestration of the facility to ensure that residents are managed efficiently by the
Well I have joined the cottage industry now. Been doing pots and those were doing...very well. Now I am busy weaving." (Mary-Resident)

“...I can cook a meal for meal for myself. I can do lot for myself...things I couldn't o...prior to being taught. I couldn't dress myself, I couldn't bath

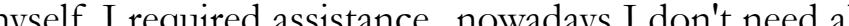
that...”(Sipho-Resident)

\section{Discussion}

The discussion describes the lived experiences of the residents according to the following quality of life indicators that were identified during the study and supported by literature. These include the influence of the physical, social and organizational environments on the residents' functional status, functional status ${ }^{8}$ which comprises physical well-being and emotional well-being and engagement in meaningful occupations ${ }^{8}$ including social interaction and relationships with other individuals.

The physical environment was identified by resident as negatively influencing the residents' independence in accessing desired areas in order to engage in their meaningful occupations or activities. As a consequence, many residents are confined to wheelchairs and are therefore restricted in their ability to propel up a steep ramp that connects the various sections of the facility.

However, the organisational environment, which consists of the routines and structures within the facility appears to pose the greatest threat to the residents' constructive engagement in activities and ability to benefit from services offered.
The professionals are those that need to hone the residual skills and abilities that residents have and refine them so that residents are able to function at an optim level. However, according to the residents and family members, the staff do not perform tasks that extend beyond their basic job description and thus appear unwilling to assist residents who experience challenges to independent mobilization.

Residents within the facility are more often than not dependent on the services provided to them by the staff. Staff and residents are in proximity with each other on a daily basis, and through the development of negative attitudes by the staff, an equally negative response by the residents is developed, as was identified by family members. Consequently, a strained relationship creates a hostile and tense environment, restricting the communication between the two involved parties.

greater part of the day, and resultantly form socially ac(the residents.

It is interesting to note that each individual perceives environmental barriers in a unique way. The results reflected that individuals using manual wheelchairs perceive the physical environment as a barrier to accessibility, whereas those in electric wheelchairs perceive the organisational environment as the barrier. A lack of resources (material, time and personnel) within the facility appears to have a significant effect on the participation in activities.

\section{Functional statu}

Occupational imbalance ${ }^{11}$ is a significant phenomenon that was identified in the residents' daily experiences. This is characterised by engagement in too much of the same activity, such as excessive sleeping and limited Staff often become so involved in their job and their exercise as was identified within the activity profiles of roles within the facility, that they subconsciously de- all the residents interviewed, as well as by the rehabilivelop a sense of detachment from the residents which tation staff of the hospital. Notwithstanding this, resiencourages them to lose focus of the ethical issues dents are provided with nursing services that cater for of their job, such as promoting autonomy and benef- those needs that contribute to physical well-being such icence. However, this negative attitude within the nurs- as provision of regular daily meals and assistance in ing staff appears to be attributed to the presence of achieving hygiene albeit limited. This is particularly im"burn-out syndrome"12 which is beyond the scope of portant for those residents within this facility who are this research. One reason for the burn-out syndrome is bed-bound and limited in their ability to independently limited human resources and has resulted in the availa- care for themselves.

ble staff over-compensating for the " to ensure the fluidity of services that residents require and deserve. In such circumstances quality of their services offered to the residents is compromised due to the over compensation and the additional responsibilities on the staff.

Additionally, the routines and "sameness" of the facility and the consistent daily schedules it encourages fosters the development of institutionalisation, not only in the residents but also in the staff. Staff appear unwilling to compromise on the established routines and organisational structures according to which they perform their duties, as was identified by the residents

"...well I tried to change (my shower days) to Tuesdays and Thursdays...So you know they are not prepared...to look at the system and re-adjust to anyone, in particular patient's desire or requirement". (Alice-Resident)

It must be acknowledged that there are nurses within the facility who perform their tasks with compassion and kindness. Nurses are with the residents for the
The residents that were interviewed have attributed their inability to perform independently in activities er than to environmental limitations. The residents that participated in the study were primarily physically impaired. The results indicated that a physical impairment is often coupled with consequent inabilities that restrict the residents' optimal participation in activities performed. Therefore, with some residents, the inability to be maximally integrated into the facility and the various activities and areas it offers, is not dominantly featured in the environmental barriers that have been identified.

\section{Emotional well-being}

Residents admitted into the facility enter as individual that have had families, jobs, interests and passions prior to their admittance. Following an injury, residents have already lost some of their physical capabilities; this is enhanced by the emotional loss of their independence in activities of daily living and placement in a chronic of daily living due to their physical impairments rath- 
As a result of the facility's organisational structures and routines, residents lack autonomy in their choice of meaningful activities and this was highlighted by family members and staff. Furthermore, the lack of control over their situations within the hospital environment is enhanced by the residents not being able to make choices regarding their fundamental requirements such as food and sleep times, which all humans are generally encouraged to select for themselves.

\section{Physical well-being}

Through their physical limitations and those the environment imposes on them, residents' individual and independent involvement in activities is hindered, therefore progressing into a culture of dependency which further restricts residents' autonomy in activity participation, thus enforcing patients to function according to the convenience of the staff who assist them to access the different departments and perform often personal and private activities of daily living, such as bathing.

The engagement of occupations within the chronic care facility cannot be fully attributed, although it contains features of, the environmental barriers, but the resident must also be given the responsibility for their occupational engagement. Residents must be able to develop positive thinking that allows for motivating conditions to develop intrinsically, thus enhancing participation in activities that are stimulating for the patients. However, the primary stimulant that encourages participation, against any or all odds, is sourced in the individual and their drive and focus for activity. This was evident in staff and family members, who identified that the desire of the residents to participate in their everyday activities, despite their limitations and those of the environment, is determined by their own levels of motivation.

\section{Engagement in meaningful occupations}

Engagement in meaningful occupations has been highlighted in literature as fundamental to health and participation in such is associated with well-being and personal satisfaction.

Occupational deprivation ${ }^{11}$ was observed within a number of residents as a result of the physical and organi- tional environments. Physical limitations that involve ent and the person with a chronic cor tion as well as organisational limitations may impact, to a certain degree, on the residents' participation in occupation within their immediate context. Confinement to a wheelchair or bed, inaccessible facilities and limited or apathetic staff are all external barriers to effective and bala sequently, the residents are not engaged in meaningful activities regularly, leading to boredom.

Deprivation may also be attributed to the decreased votition that was observed in certain residents which is an important contributor to engagement in occupation. This apathy is a consequence of learned helplessness and its associated phenomenon institutionalisation ${ }^{13}$ and fosters an external locus of control within the residents.

Unchanging and consistent service provision is limited and resultantly so is gradual functional improvement. This phenomenon, in effect, actually serves to hinde residents' well-being and challenges the ethical implications that are associated with service provision.

Residents within the facility are often admitted to the hospital due to poor family structure and/or a lack of skilled caregivers to sufficiently care for residents. Such esidents require the assistance of external individuals, such as their friends and their family, to provide them with the leverage to participate in desired activities that extend beyond the boundaries of the facility. Subsequently, the social environment, in terms of the residents' family and friends, is often restricted due to cost associated with accessing the facility, as well as the fact that individuals become so involved in their own daily lives.

This restricted family contact may additionally limtheir understanding and awareness of the reality of their relative's experience of life within the facility and thus advocacy on the part of the family for their relative's well-being is hindered.

Through the development or attainment of a physical disability or impairment, the physical functional statu of these individuals is compromised, which is exacerbated by the contextual factors which make up the hospital environment. Consequently, their engagement in meaningful activities is limited, whether due to external, environmental or internal, emotional factors found within the residents themselves.
The authors aimed to describe the lived experiences of residents residing within a chronic care facility through a phenomenological lens. The findings illustrated that many of the residents' quality of life is compromised, primarily due to the environment's influence on their functional status and the resultant lack of engagement in meaningful and valued occupations. Consequently, their lives are often and predominantly associated to phenomena such as occupational deprivation and imbalance; learned helplessness and reduced autonomy Each phenomenon perpetuates and thrives off each other, which further impedes the quality of life of the residents at the institution. As identified, the limited resources, perceptions of nurses attitudes and routine aggravate the levels of dependence and control the residents have over their lives.

This further impedes the residents' optimal and autonomous functioning and engagement in meaningful occupations within the facility.

Notwithstanding this, it must also be considered that the routines of the facility are established to maintain a sense of control and fluent management of the facility. Through the use of the above-mentioned quality of life indicators, it can be concluded that a number of residents within the hospital experience a diminished quality of life. Consequently, elements that make up life within the facility; being both promotors and inhibitors; either perpetuate the challenges imposed on the residents and the staff, or enhance some of the residents' and staffs' lives.

This calls for the urgent development and implementation of a chronic care policy within South Africa, which will provide guidelines for service provision withi chronic care facilities. This will ensure that good prac tice principles are implemented in order to facilitate and attain an improved quality of life for residents of such facilities.

\section{Limitations}

Limitations within this study may have included the following: The acquisition of unbiased testimonials may be compromised due to participants difficulty in precisely recalling their experiences as well as their reservation displayed; the reliability of the family members' testimonial may be questionable due to their limited contact with the residents and lastly, the findings from his case study are not generalisable to other chronic facilities due to the phenomenological nature of the study.

\section{Acknowledgement}

The authors would like to acknowledge Ms N. Motala for her co-supervision of this project.

\section{References}

1. Van Manen M. Researching lived experience: human science for an action sensitive pedagogy. The Althous Press. University of Western Ontario. Ontario. Canada, 1990.

2. Stamm T, Lovelock L, Stew G, Nell V, Smolen J, Machold K, Jonsson H, Sadlo G. I Have a Disease, but Am Not ill: A Narrative Study of Occupational Balance in People with Rheumatoid Arthritis. Occupational Therapy Journal of Research: Occupation, Participation and Health 2007:29(1):33-38

3. Yuen HK, Burik JK, Smith TG. Impact of participation in volunteer activities for residents living in longterm facilities. American Journal of Occupational Therapy 2008:62:71-76.

4. World Health Organisation. Ethical choices in longterm care: What does justice require? 2002. http:// www.who.int/mediacentre/notes/ethical_choices.pdf (Accessed 07/06/2010).

5. Patients' Rights Charter. http://www.doh.gov.za/ docs/legislation/patientsright/charter.html. (Accessed 05/03/2014

6. Batho Pele Policy. http://www.kznhealth.gov.za/bathopele.htm. (Accessed 05/03/2014)

7. Hasselkus BR. Eleanor Clarke Slagle Lecture-The world of everyday occupation: Real people, real lives. American Journal of Occupational Therapy 2006:60(6):637640.

8. Carr AJ, Thompson PW, Kirwan JR. Quality of Life Measures. British Journal of Rheumatology 1996:35:275281.

9. Singh DA. Effective Management of Long Term Care Facilities. Sudbury. Jones and Bartlett Publishers, 2005.

0. McKay E, Molineux M. Occupation: Reaffirming its Place in our Practice. British Journal of Occupational Therapy 2000:63(5):241-242.

11. Wilcock AA. Occupation for Health. British Journal of Occupational Therapy 1998:61(8):340-345.

12. Poncet MC, Toullic P, Papazian L, Kentish-Barnes 
N, Timsit JF, Pochard F, Azoulay E. Burnout syndrome in critical care nursing staff. American journal of respiratory and critical care medicine 2007:175(7):698-704.
13. Venter E, Zietsman K. Rehabilitation of the mentally ill in long-term institutionalisation IN R Crouch, V Alers (2005) Occupational Therapy in Psychiatry and Mental Health (4th edition). London: Whurr Publishers Limited. 